\title{
fouille clouée de grande profondeur (usine E.D.F. de Ferrières-sur-Ariège)
}

\author{
BLONDEAU Francis \\ Directeur Général Adjoint TERRASOL* \\ LEDEUIL Emile \\ Ingénieur Principal E.D.F.**
}

\section{INTRODUCTION}

\subsection{Description des ouvrages}

Le site de Ferrières se trouve dans la partie centrale du département de l'Ariège, à l'amont immédiat de la ville de Foix

La chute comprend un barrage poids en béton de $40 \mathrm{~m}$ de hauteur, une galerie de $4,7 \mathrm{~km}$ arrivant à une usine semi-enterrée, objet de cette étude.

Sur une terrasse alluvio-glaciaire, a une altitude de $409 \mathrm{~m}$ recouvrant un substratum rocheux oscillant entre 401 et 403, la fouille de l'usine à réaliser descend à la cote 383 et localement à 377 , c'est-à-dire à $30 \mathrm{~m}$ environ (fig. 1 ).

\subsection{Description géologique}

Les sondages effectués indiquent qu'en rive gauche se trouve un ancien lit ayant surcreusé le bed-rock jusqu'à une cote 393, l'usine est donc implantée sur un plateau de schistes paléozoïques noirs assez fortement broyés.
Ces schistes sont recouverts d'un mélange d'alluvions et de moraines glacières comportant de gros blocs de granite.

La cote moyenne de l'Ariège devant l'usine est de 391, pouvant monter à 394 (crue décennale) ou même 400 en crue millénale du projet. La présence du lit ancien limite donc la hauteur piézométrique à la hauteur de l'Ariège. Le substraturn schisteux peut être considéré comme étanche, aux fissures près.

\subsection{Choix des principes de fouilles}

Dans les moraines la fouille est descendue en talus à 1 pour 1 jusqu'à une risberme de $3 \mathrm{~m}$ située à la cote 400 (fig. 2).

Dans les schistes l'économie du projet prévoit une fouille verticale et des bétons de soutènement classiques.

* 52, quai de Dion-Bouton, 92806 Puteaux.

** Région d'Equipement Alpes-Marseille, 140, avenue Viton, B.P. 560,13401 Marseille. 


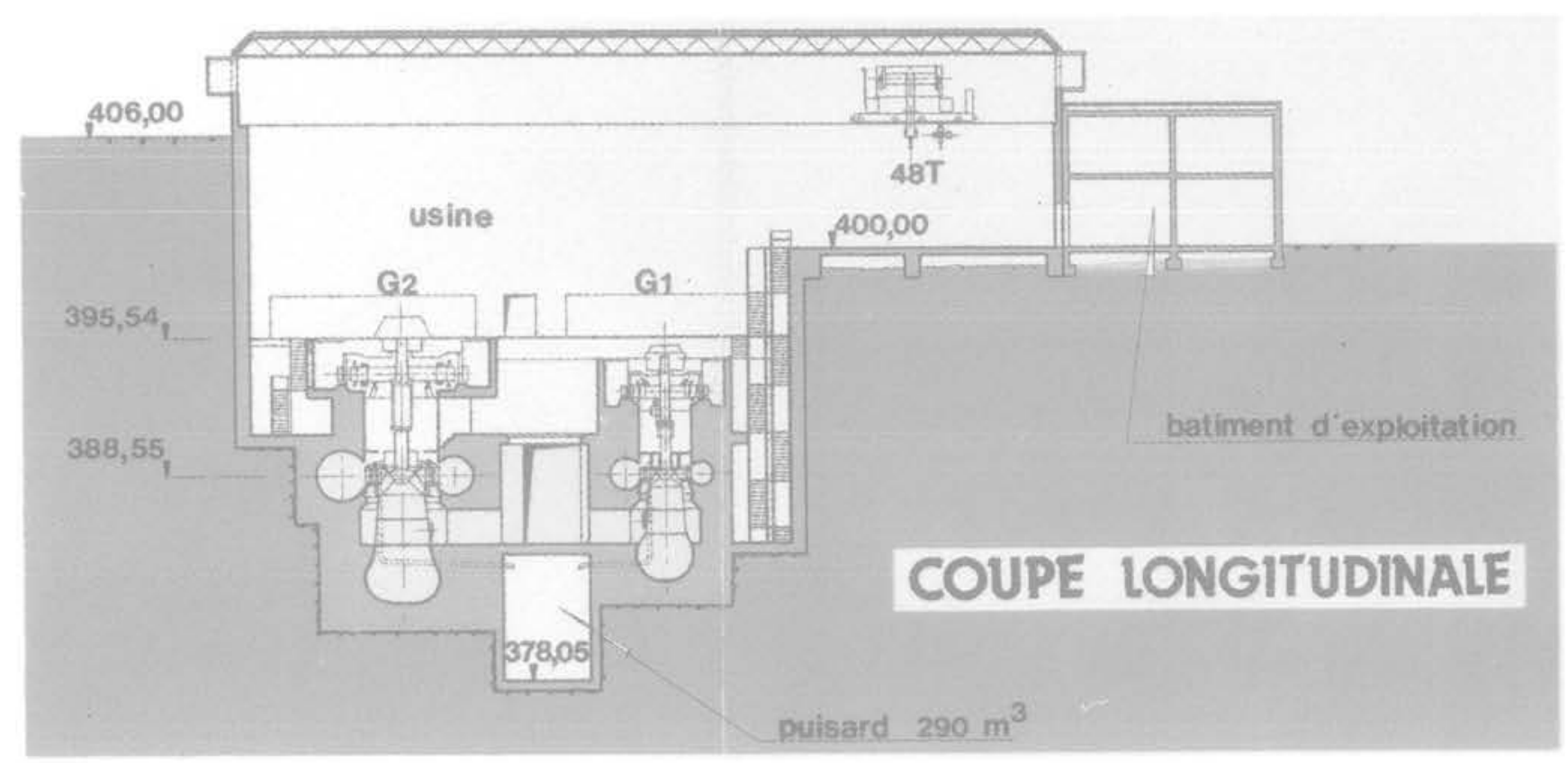

Fig. 1. - Coupe de l'usine de Ferrières-sur-Ariège.

L'aspect très broyé du matériau schisteux, la qualité d'écrasement des grains très variable allant jusqu'à l'écrasement entre les doigts et les plans de schistosité à $45^{\circ}$, très défavorable en rive gauche, obligent à un clouage de la totalité des parois.

La fouille devant être drainée en surface, un voile d'injection est réalisé tout autour entre 10 et $15 \mathrm{~m}$ de distance de l'usine et jusqu'à la cote 370 (voile mono-linéaire avec $3 \mathrm{~m}$ d'espacement et injections en quinconce).

La fouille est réalisée par passes successives de $2 \mathrm{~m}$ afin d'éviter tout échafaudage pour les ancrages et garantir la stabilité à court terme. La première passe de $2 \mathrm{~m}$ consiste à mettre en ceuvre une poutre de ceinture en béton armé formant un angle avec un retour de $1,40 \mathrm{~m}$. Les phases suivantes de $2 \mathrm{~m}$ reçoivent, avant toute continuation, un réseau de drainage par forages et rouleaux drainant en surface, des ancrages décrits ci-après, un treillis soudé et $10 \mathrm{~cm}$ de béton projeté.

L'excavation par phases de $2 \mathrm{~m}$ est précédée par un pré-découpage du rocher (espacement $0,40 \mathrm{~m}$, cordon de $70 \mathrm{~g}$ dans un trou de diamètre $\varnothing 64 \mathrm{~mm})$. Ce pré-découpage est fait sur $10 \mathrm{~m}$ maximum de profondeur. Le gros diamètre garantissant $15 \mathrm{~cm}$ de déviation maximum, il a été nécessaire de décaler le plan de pré-découpage de $0,60 \mathrm{~m}$ pour la deuxième phase faite à partir de 390 .

Pour l'abattage, les trous (espacement $2,50 \mathrm{~m}$, profondeur $2,50 \mathrm{~m}$ ) ne sont chargés que sur le mètre du fond avec $1,5 \mathrm{~kg}$ d'explosif.

L'arrivée des conduites forcées $\varnothing 5 \mathrm{~m}$ exécutée en sous-terrain est réalisée par des anneaux en béton armé ancrés dans le rocher afin que la fouille passe juste devant sans risquer la stabilité de l'ensemble.

Le plan de fouille permet un chantier tournant abattage, chargement, ancrages, drainages, béton projeté et un rythme de $2 \mathrm{~m}$ par semaine a été pratiquement soutenu.

\section{JUSTIFICATION}

\subsection{Contexte géotechnique relatif au clouage}

Les schistes paléozoïques constituant le matériau d'environnement de la fouille de l'usine ont, à cet endroit, un pendage de $45^{\circ}$ environ, de la rive gauche vers la rive droite (fig. 2).

Les parois de la fouille sont de ce fait très dissymétriques si l'on considère le point de vue du soutènement à mettre en œuvre.

Aucun essai géotechnique n'étant disponible pour le dimensionnement du clouage, nous nous sommes calés sur l'observation d'un petit glissement survenu lors d'un pré-terrassement pour le pont sur le CD8 en décembre 1981. (fig. 3). Ce glissement typique d'un glissement banc sur banc dans un schiste, nous a amené à prendre comme caractéristiques du matériau: $\gamma=20 \mathrm{kN} / \mathrm{m}^{3}$

$\mathrm{C}=0 \quad \phi=45^{\circ}$ dans le plan de schistosité $\mathrm{C}=40 \mathrm{kPa} \quad \phi=45^{\circ}$ dans la masse

La valeur de la cohésion prise en compte est totalement arbitraire dans le cas présent. Ceci n'est pas très 


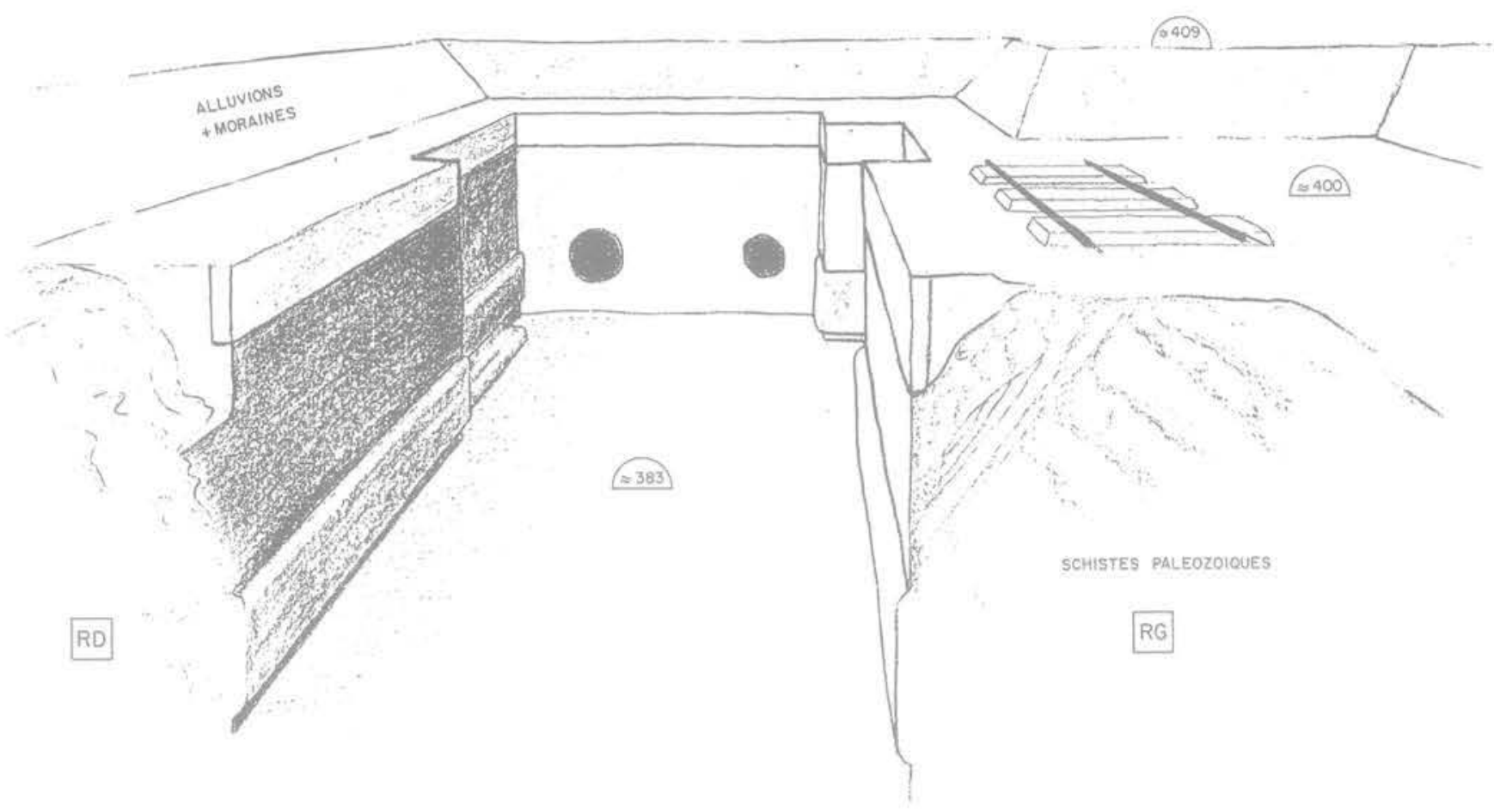

Fig. 2. - Vue schématique des terrassements.

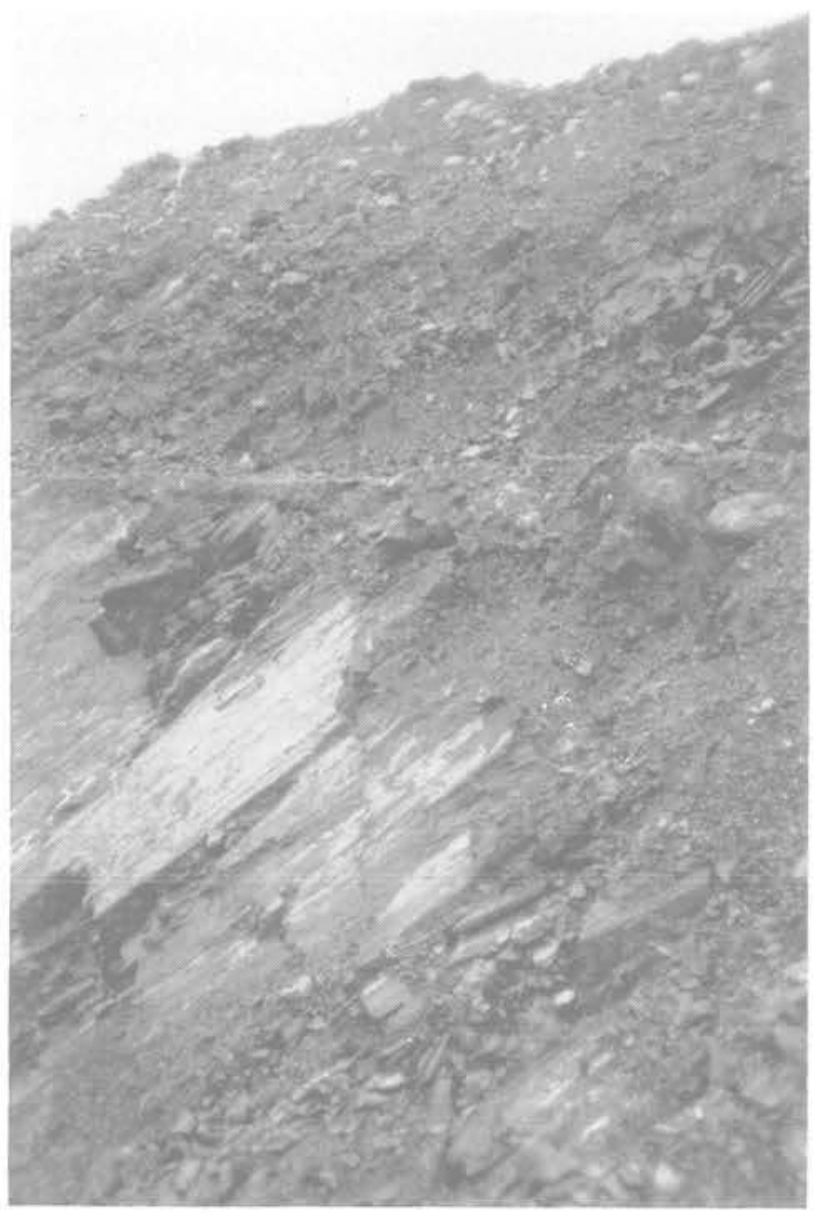

Fig. 3a. - Glissement survenu dans les schistes au voisinage de la fouille de l'usine.
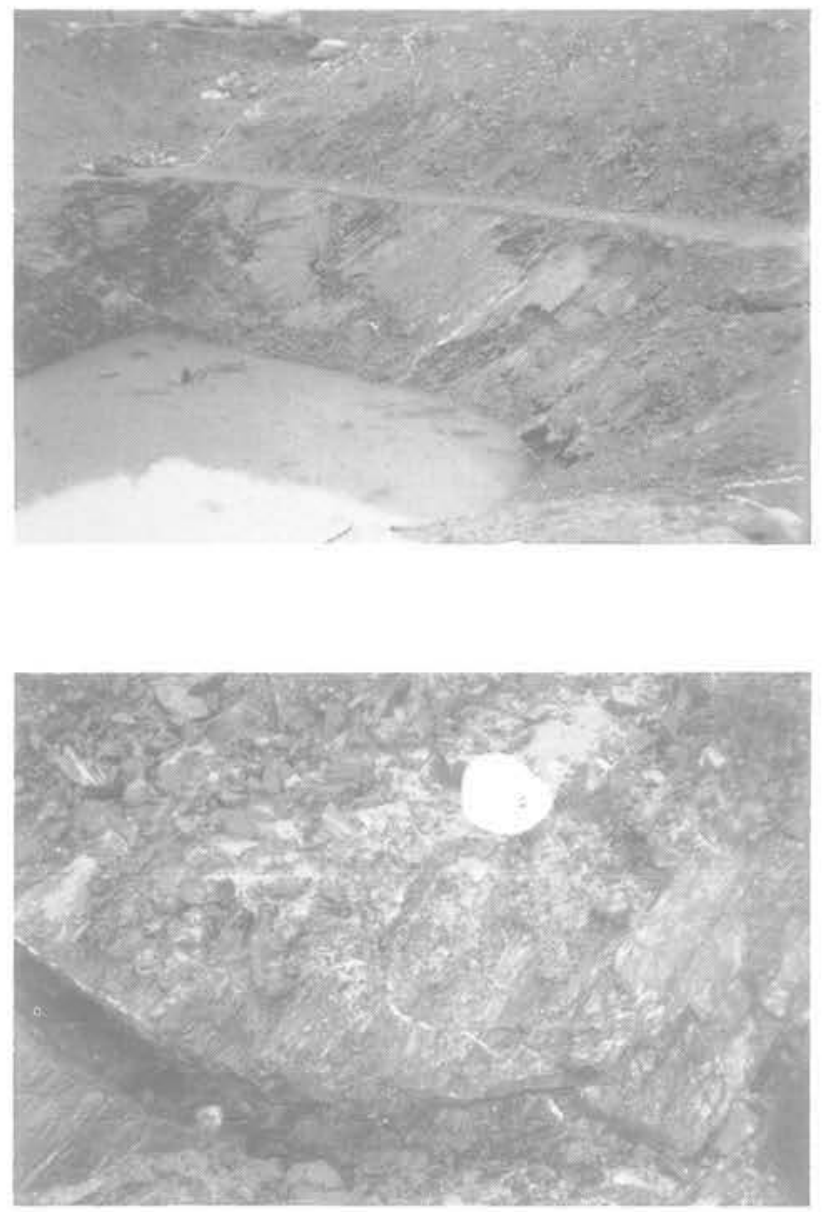

Fig. 3b. - Détail de la rupture. 
gênant car, pour le talus rive gauche qui est le plus critique, les surfaces de rupture coincident avec les plans de schistosité, où la cohésion est prise nulle.

Comme on l'a indiqué précédemment, un écran d'étanchéité avait été mis en place à l'extérieur de la fouille afin d'éviter un éventuel traitement d'interception des cheminements d'eau contradictoire avec l'existence des drains prévus en parement de la fouille et exécutés tous les $2 \mathrm{~m}$ au fur et à mesure de l'avance des terrassements.

De ce fait, aucune pression interstitielle n'a été prise en compte dans les calculs.

\subsection{Logiciel TALREN}

Le logiciel de calcul est le programme TALREN (Talus Renforcés) mis au point par TERRASOL, dont on rappelle ici les principales caractéristiques.

Le calcul est un calcul à la rupture le long d'une surface de rupture potentielle (plan, cercle, autre courbe...) traversée par les renforcements: barres passives, tirants précontraints (fig. 4).
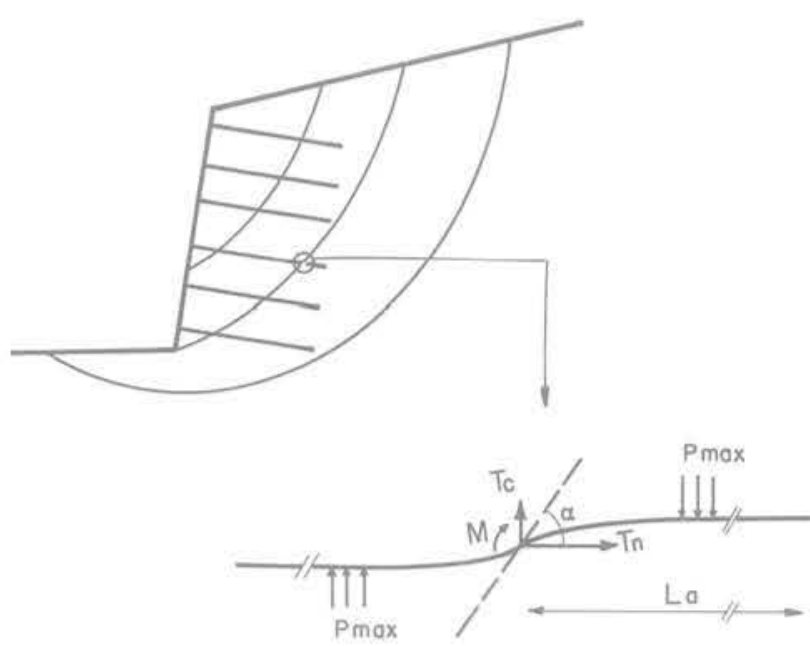

Fig. 4. - Paramètres utilisés pour la prise en compte des renforcements dans le logiciel TALREN.

Quatre critères sont à vérifier:

2.2.1. Résistance de la barre: les contraintes dans l'acier doivent être inférieures aux contraintes maximales mobilisables:

- s'il est fait abstraction du cisaillement sol-barre, on vérifie que l'effort de traction $T_{n}$ est tel que $T_{n} \leqslant \sigma_{e} \times S$ $\left(\sigma_{e}\right.$ limite élastique de l'acier, $\mathrm{S}$ section de la barre);

- si l'on souhaite prendre en compte traction et cisaillement, un calcul basé sur le principe du travail maximal permet de déterminer les valeurs de la traction $T_{n}$ et du cisaillement $T_{c}$ mobilisables en fonction de l'angle $\alpha$ que fait la surface de rupture avec l'inclusion.
2.2.2. Frottement sol-barres: il faut vérifier que:

$\mathrm{T}_{\mathrm{n}} \leqslant \mathrm{T}_{\mathrm{nl}}$

où: $\mathrm{T}_{\mathrm{nl}}=\mathrm{B} \cdot \tau_{\ell} \cdot \mathrm{L}_{\mathrm{a}}=\mathrm{F} \cdot \mathrm{L}_{\mathrm{a}}$

avec: $\mathrm{B}$ diamètre de l'inclusion

$\mathrm{L}_{\mathrm{a}}$ longueur d'inclusion en arrière de la surface de rupture

$\tau_{f}$ frottement unitaire limite

F frottement limite par mètre linéaire de barre

2.2.3. Réaction normale sol-barres: il faut vérifier que l'effort de cisaillement mobilisable dans l'inclusion peut être équilibré par la réaction du sol. Si l'on assimile l'inclusion à un pieu soumis à des efforts transversaux, on démontre que l'effort tranchant maximal mobilisable dans l'inclusion supposée infiniment longue par rapport à la longueur de transfert est:

$\mathrm{T}_{\mathrm{c}}=\frac{\mathrm{B} \ell_{\mathrm{o}}}{2} \mathrm{p}_{\max }$

avec: $\mathrm{B}$ diamêtre de l'inclusion

$\ell_{\mathrm{o}}$ longueur de transfert $=\sqrt[4]{\frac{4 \mathrm{EI}}{\mathrm{k}_{\mathrm{s}} \mathrm{B}}}$

El rigidité transversale de l'inclusion

$\mathrm{k}_{\mathrm{s}} \mathrm{B}$ module de réaction horizontale sol-clou

$\mathrm{p}_{\max }$ réaction latérale mobilisée par le sol, que

l'on prend en général égale à la pression de fluage pressiométrique

2.2.4. Résistance au cisaillement du sol: on vérifie que, le long de la surface de rupture, les contraintes de cisaillement au sein du sol sont inférieures à la résistance au cisaillement de celui-ci:

$\tau \leqslant \mathrm{C}+\sigma \operatorname{tg} \phi$

avec: $\mathrm{C}$ cohésion

$\sigma$ contrainte normale

$\phi$ angle de frottement interne

Les coefficients de sécurité sont appliqués aux différents constituants du mur cloué:

- Sol:

Selon les cas, on adopte $F_{\mathrm{s}}=1,3$ à 1,5 en réduisant la résistance par:

$$
\mathrm{C}^{*}=\frac{\mathrm{C}}{\mathrm{F}_{\mathrm{s}}} \quad \operatorname{tg} \phi^{*}=\frac{\operatorname{tg} \phi}{\mathrm{F}_{\mathrm{s}}}
$$

- Renforcement:

La traction de service $T_{n}$ est limitée à une fraction de la traction limite élastique $T_{G}$ par:

$\mathrm{T}_{\mathrm{n}}=\frac{\mathrm{T}_{\mathrm{G}}}{\mathrm{F}_{\mathrm{R}}}$

avec:

$F_{R}=\frac{4}{3}$ ou $\frac{3}{2}$

selon le cas (provisoire ou définitif).

- Interaction sol-renforcement

Le frottement limite sol-barre est affecté par le programme du même coefficient de sécurité que la résistance au cisaillement du sol.

Lorsque le cisaillement est pris en compte, la réaction normale sol-barre, limitée à la pression de fluage $\mathrm{p}_{f}$, n'est pas affectée de coefficient de sécurité. 
On notera que cette façon de procéder cumule les coefficients de sécurité et qu'il y aurait normalement lieu de les modifier dans le sens d'une réduction si l'on admettait une approche moins conservatrice.

Le programme TALREN utilise deux méthodes de calcul:

- bishop simplifiée en rupture circulaire,

- perturbations en rupture non circulaire.

\subsection{Hypothèses propres à Ferrières}

Dans le cas présent, nous n'avons pris en compte que la traction des barres, sans cisaillement.

Le frottement sol-barre était défini par sa valeur limite:

$$
\tau_{\mathrm{lim}}=350 \mathrm{kPa}
$$

déduites des abaques de Bustamante et Gianeselli (fig. 5) pour p $\ell$ compris entre 4 et $5 \mathrm{MPa}$ et confirmée par les observations faites par TERRASOL sur différents sites de clouage intéressant des matériaux frottants à plus de $45^{\circ}$.

Une surcharge de $50 \mathrm{kPa}$ simule les engins de manutention.

En rive gauche les talus de moraine étaient repoussés à une quinzaine de mètres de la fouille et à 4 ou $5 \mathrm{~m}$ en rive droite. Leur présence n'affectait pas a priori la stabilité générale de la fouille dont la hauteur pratique, en zone courante, était ainsi ramenée à $17 \mathrm{~m}$.
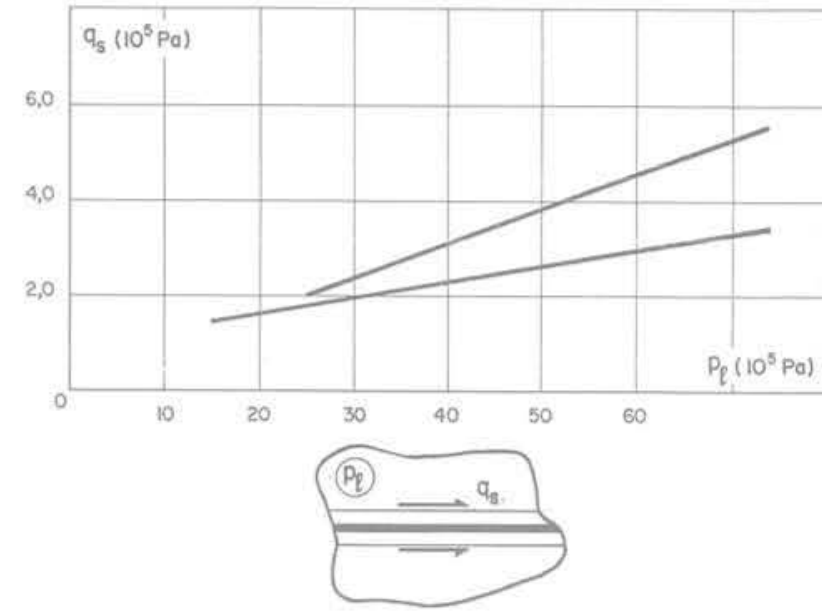

Fig. 5. - Extrait des abaques de BustamanteGianeselli relatifs au frottement sol-inclusion.

\subsection{Dimensionnement du clouage}

Un premier dimensionnement fait avec des inclusions de relativement forte capacité $\left(T_{G}=300\right.$ à $600 \mathrm{kN}$ selon les endroits) a abouti au schéma de clouage présenté figure 6. Sur cette figure les clous en traits pleins correspondent au profil typique de la rive droite. L'extension en pointillés correspond à la rive gauche où le pendage est défavorable.
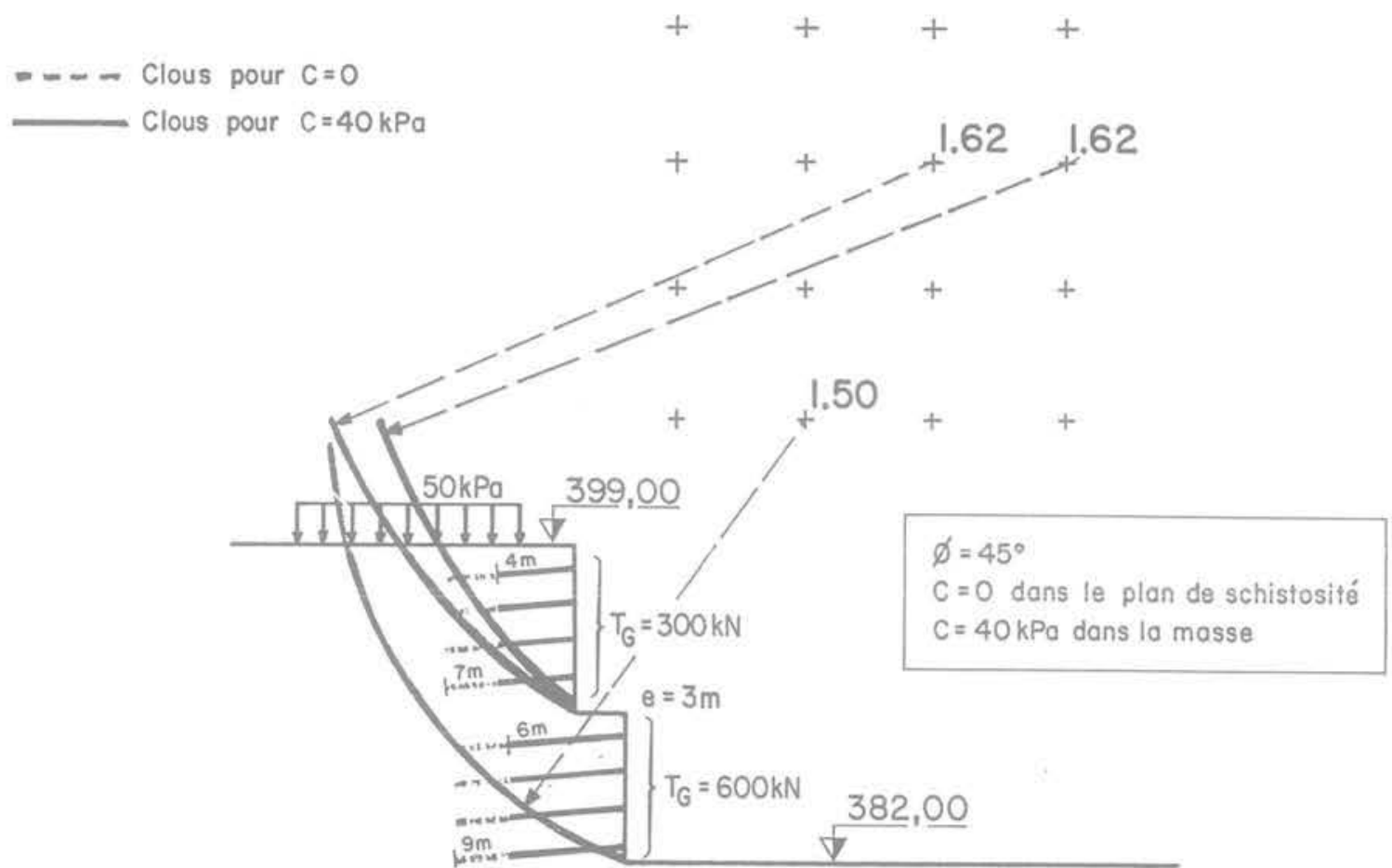

Fig. 6. - Premier schéma de clouage envisagé pour la fouille de l'usine. 


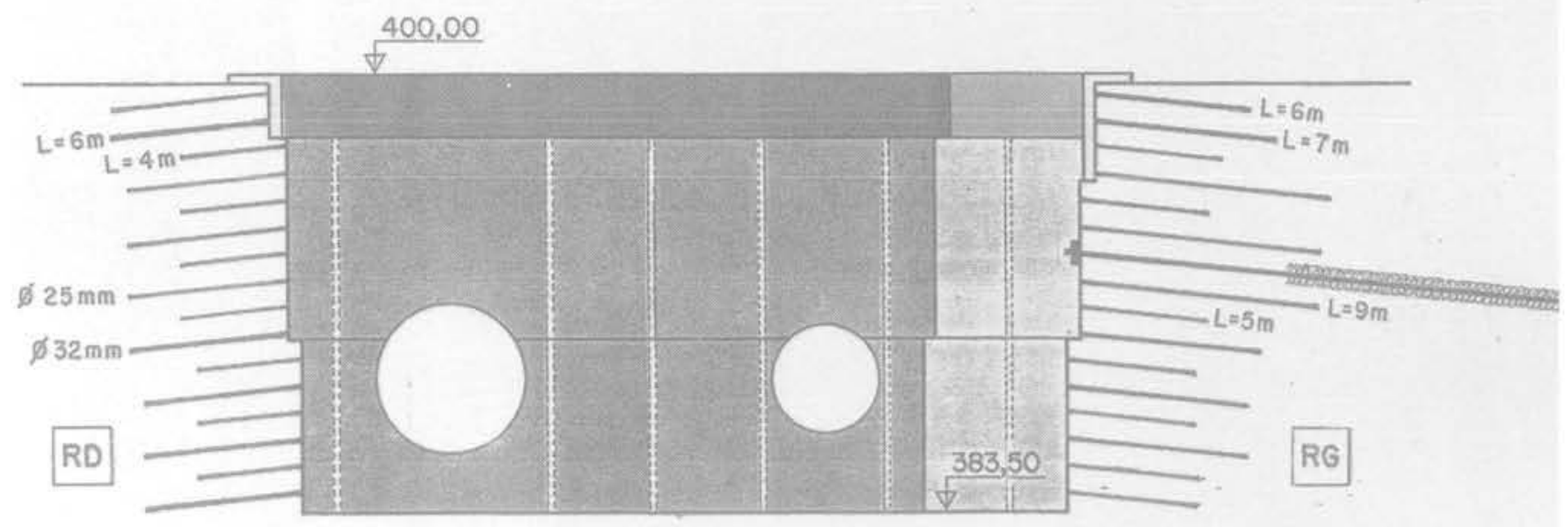

Fig. 7. - Schéma de clouage effectivement retenu.

Le maillage auquel aboutissait ce premier parti était de $6 \mathrm{~m}^{2}$ par clou.

A la demande d'E.D.F. qui souhaitait mettre en ceuvre des clous de plus faible capacité et plus longs, un maillage plus dense a été étudié; les barres retenues étaient des aciers HA:

$$
\begin{array}{ll}
\varnothing 32 \mathrm{~mm} & \mathrm{~T}_{\mathrm{G}}=338 \mathrm{kN} \\
\varnothing 25 \mathrm{~mm} & \mathrm{~T}_{\mathrm{G}}=206 \mathrm{kN}
\end{array}
$$

travaillant aux $2 / 3$ de la limite élastique.

Le coefficient de sécurité du sol a été réduit à $\mathrm{F}=1,3$.

Le schéma de clouage correspondant apparaît figure 7.

La densité du maillage est double de la précédente.

On notera que le calcul conduit, comme pratiquement toujours en pareil cas, à une longueur de clous relativement faible en tête. L'on sait maintenant par expérience, et le cas de Ferrières a contribué à cette réflexion, que ces longueurs théoriques sont trop courtes et qu'en pratique les deux premiers lits de clous devraient avoir une longueur se rapprochant de la hauteur du talus définitif. Ceci permet de constituer une sorte de dalle qui limite très sensiblement les mouvements de décompression en surface, renvoyant ainsi en profondeur les déformations horizontales nécessaires à la mise en traction des clous. Les tirants mentionnés en rive gauche ont été ajoutés à la suite de l'incident développé au point 3.

\subsection{Réflexions sur le scellement}

En pratique, les barres ont été placées dans des forages $\varnothing 56 \mathrm{~mm}$, conduisant à une résistance théorique de scellement:

$$
\mathrm{f}=350 \times \pi \times 0,056=62 \mathrm{kN} / \mathrm{ml}
$$

Un essai d'arrachement (fig. 8) a êté fait sur une des barres en place $\left(\varnothing 32 \mathrm{~mm} ; \mathrm{T}_{\mathrm{G}}=338 \mathrm{kN} ; \mathrm{L}=6 \mathrm{~m}\right)$. La barre s'est rompue au filetage de la tête pour une

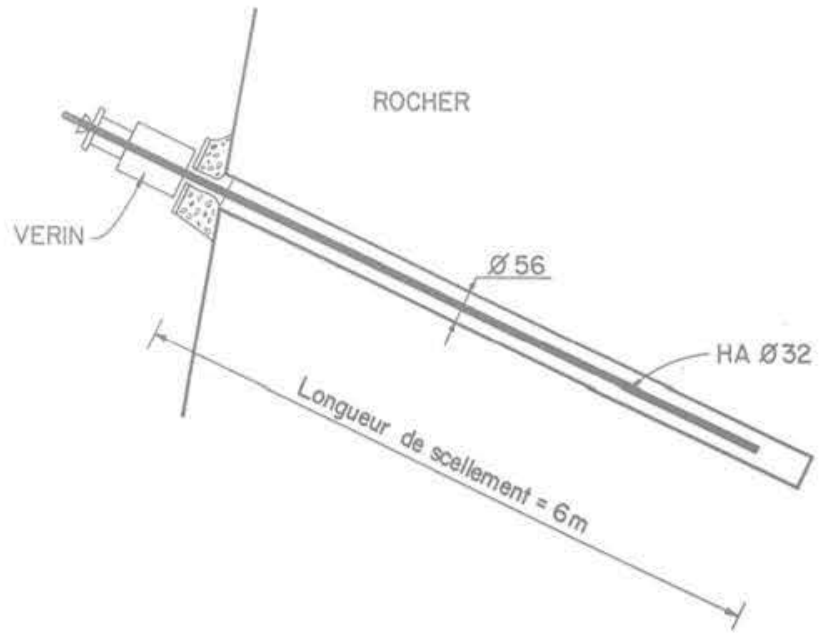

Fig. 8. - Schéma de l'essai d'arrachement pratiqué in situ.

traction de $334 \mathrm{kN}$. La résistance au scellement était donc telle que:

$$
\begin{aligned}
& f \geqslant \frac{334}{6} \\
& f \geqslant 56 \mathrm{kN} / \mathrm{ml}
\end{aligned}
$$

Cette valeur est proche de la valeur théorique de calcul. Il aurait cependant été intéressant d'obtenir la valeur limite de l'adhérence en testant une longueur de scellement plus courte ( $2 \mathrm{~m}$ par exemple).

\section{L'INCIDENT}

Le dispositif d'auscultation prévu pour le suivi de la fouille et indispensable à une bonne sécurité ne fut installé qu'avec un certain retard, alors que la fouille 


\section{RIVE GAUCHE Niveau 400 NGF}

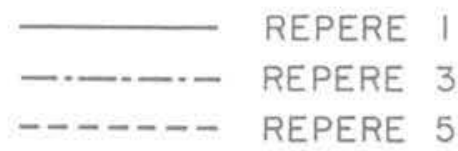

\section{RIVE DROITE Niveau 400 NGF}

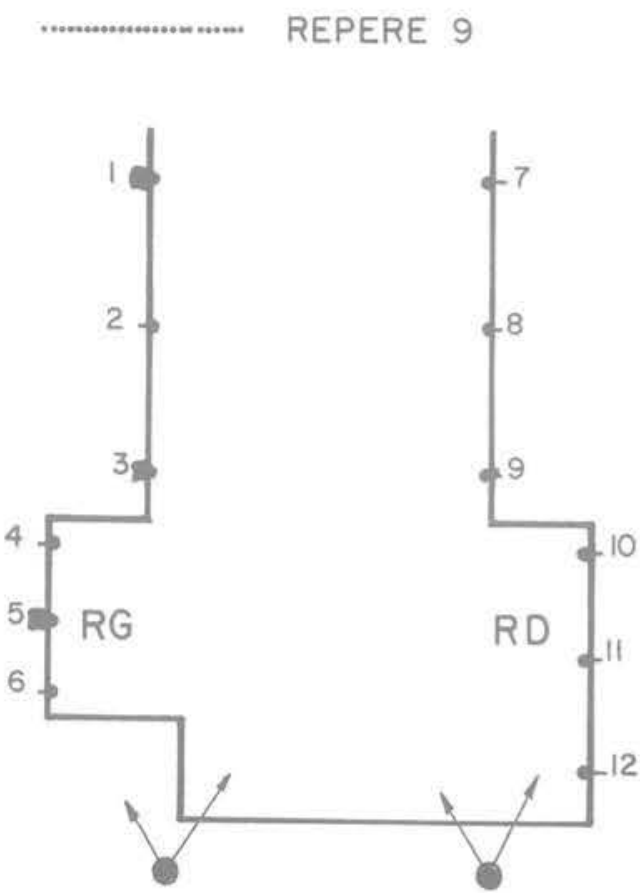

Piliers de visée

Fig. 9. - Implantation du dispositif d'auscultation par topométrie sur le couronnement de la fouille.

atteignait la cote 392. Il était constitué de 9 points sur la poutre de ceinture visés à partir de deux pilliers (fig. 9), L'excavation s'était déroulée sans problèmes jusqu'alors.

A peine les premières mesures effectuées, une fissure apparut en rive gauche à une quinzaine de mètres en arrière du bord de la fouille (fig. 10) accompagnée de craquements. L'extrémité libre de la poutre s'est déplacée de $5 \mathrm{~mm}$ vers la fouille: repère 1 (fig. 11).

Bien que théoriquement il n'y avait pas à craindre de désordre majeur à la suite de cet "incident», il fut immédiatement décidé de:

- reboucher la fissure au mieux et de favoriser l'écoulement des eaux en surface afin d'éviter toute mise en charge de la fissure;

- assurer un complément de stabilité du massif par mise en place de 10 tirants dans la fouille à la cote 393 environ, plus 4 tirants pour accrocher le massif situé sur la plate-forme.

Les tirants font $1000 \mathrm{kN}$ en service, $20 \mathrm{~m}$ de long dont $12 \mathrm{~m}$ scellés. Les têtes sont reliées par une lierne

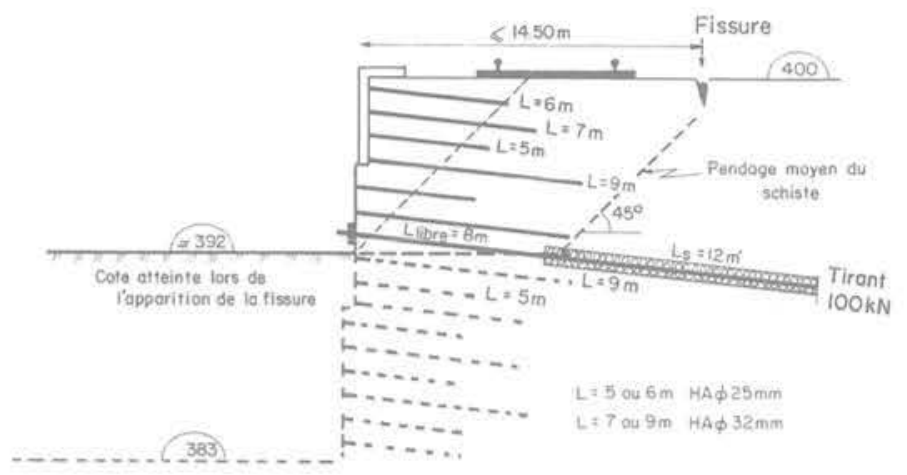

Fig. 10. - Coupe de la fouille lors de l'apparition de la fissure en arrière de la voie de grue.

de continuité en H.E.B. 300 bloquée par béton au radier.

Les terrassements se sont poursuivis sans aucun incident jusqu'à la fin de cette phase de chantier.

\section{AUSCULTATION}

La figure 11 montre l'évolution des déplacements observés depuis les deux piliers.

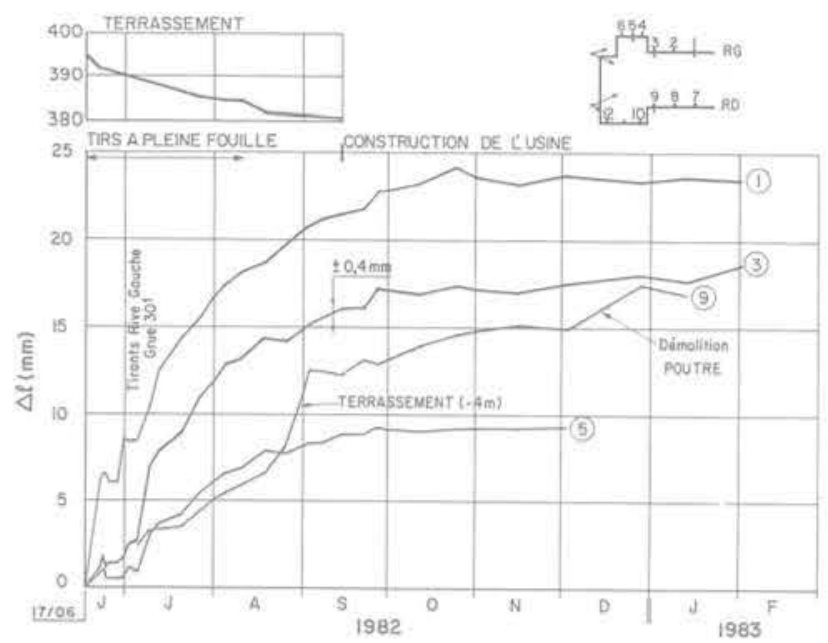

Fig. 11. - Evolution des principaux témoins en tête de fouille lors des terrassements.

Le déplacement maximum mesuré en extrémité de poutre (repère 1) a été de $25 \mathrm{~mm}$ après mise en place de l'auscultation; il convient d'y ajouter le déplacement antérieur qui a pu être de l'ordre de 8 à $10 \mathrm{~mm}$ la fouille faisant environ $8 \mathrm{~m}$ au moment des premières mesures. Cela conduit à un déplacement total de l'ordre de $35 \mathrm{~mm}$, soit $2 \%$ de la hauteur de la fouille.

La stabilisation des mouvements est atteinte pratiquement à la fin de la période de terrassement, début octobre 1982, sauf pour le repère 9 qui subit un déplacement d'environ $1 \mathrm{~mm}$ par mois jusqu'à la 
montée de la structure de l'usine (les terrassements dans cette zone étaient décalés par rapport aux terrassements dans la zone amont).

On notera la bonne précision de la mesure, de l'ordre de $\pm 0,4 \mathrm{~mm}$ sur les déplacements horizontaux.

Le pivotement de la poutre rive gauche, encastrée côté amont et libre côté aval, s'est confirmé tout au long du terrassement.

On ne dispose malheureusement pas de mesures de déplacement à des niveaux intermédiaires du mur qui auraient permis de compléter l'information sur le comportement de celui-ci le long d'une section verticale.

\section{INTERPRÉTATION - COMMENTAIRES}

L'origine de la fissuration observée est liée à la longueur relativement courte des barres de tête. La fissure est en effet apparue au-delà de l'extrémité des lits supérieurs et correspond à la phase de mise en tension des barres inférieures inhérante au mode de fonctionnement de l'ouvrage.

Il n'y a pas eu de rupture des barres, car il y aurait eu alors un effondrement brutal de la structure. Un calcul de stabilité refait a posteriori montre d'ailleurs qu'en faisant travailler l'acier à sa limite élastique, le coefficient de sécurité sur le sol et sur le scellement est supérieur à 1,8 pour la fouille à la cote 392 .

Le mur s'est conduit comme un mur poids à l'avant de la fissure.

Les tirants ont certainement limité le développement du mouvement à la cote 393 , la crête de la fouille a cependant continué à se déplacer. Ils ont eu, à notre sens, un intérêt essentiellement psychologique vis-à-vis du personnel de chantier amené à travailler au pied de la fouille.
Le déplacement rive gauche s'est certainement trouvé accentué par le fait que l'effet des explosifs utilisés pour le pré-découpage se faisait beaucoup plus sentir en rive gauche ou la schistosité guide les ondes vers la surface, alors qu'en rive droite elle a tendance à les enfouir. Des observations faites sur le chantier ont montré que les tirs étaient sensibles à plus de $200 \mathrm{~m}$ de la fouille en rive gauche, alors qu'ils n'étaient pratiquement pas ressentis en-dehors des abords immédiats en rive droite.

Les vibrations ont ainsi certainement eu tendance à provoquer un décollement des bancs rive gauche. On notera que, vis-à-vis de cet effet, le clouage constitue un confortement très efficace car il joue le même rôle que les coutures dans le béton armé vis-à-vis de la fissuration.

\section{CONCLUSION}

Il s'agit d'une fouille importante. La solution adoptée conduisait à des volumes de béton et de terrassements beaucoup plus faibles que pour une solution mur poids. Le soin apporté par l'entreprise à sa réalisation a permis de limiter les hors-profils et de tenir pratiquement les quantités théoriques, ce quí doit être salué compte tenu de la difficulté de ce chantier.

Actuellement (janvier 1984) le gros-œuvre de l'usine est pratiquement terminé.

\section{BIBLIOGRAPHIE}

BUSTAMANTE, M. ; GIANESELLI, G. (1981). Prévision de la capacité portante des pieux isolés sous charge verticale. Bulletin de liaison des Laboratoires des Ponts et Chaussées, $n^{\circ} 113$, pages 83 à 108. 